\title{
Keyword Index Volume 22 (2014)
}

$\begin{array}{lrlr}\text { 1/f sound } & 419 & \text { biomedical signal processing } & 651 \\ \text { abandonment } & & \text { bio-sensing computing } & 387 \\ \text { absence } & 443 & \text { blood loss } & 901 \\ \text { accelerometry } & 369 & \text { body image } & 157 \\ \text { acceptability } & 199 & \text { Body Mass Index (BMI) } & 403 \\ \text { accident } & 689 & \text { bone drilling } & 243,741 \\ \text { acute pain } & 369 & \text { bowed legs } & 325 \\ \text { Adaptive Neuro-Fuzzy Inference System } & 63 & \text { breast thickness } & 345 \\ \text { aged care } & 835 & & \\ \text { agentroller } & 99 & \text { capacitive sensor } & 867 \\ \text { allogenic blood transfusions } & 561 & \text { cardiac surgery } & 317 \\ \text { amputation } & 123 & \text { cardiogenic oscillations } & 717 \\ \text { angular stable } & 157 & \text { cardiomyocytes } & 37 \\ \text { ant colony } & 909 & \text { cardiopulmonary performance testing } & 179 \\ \text { AnyBody } & 775 & \text { cardiopulmonary resuscitation } & 351 \\ \text { application } & 627 & \text { CAS } & 115 \\ \text { art and music therapy } & 351 & \text { CBIR } & 13 \\ \text { arthroplasty } & 453 & \text { cell phone } & 597 \\ \text { artificial bee colony (ABC) } & 129 & \text { cerebral aneurysm } & 209 \\ \text { artificial limb } & 775 & \text { chair-rise pattern } & 627 \\ \text { aseptic loosening } & 157 & \text { checklists } & 795 \\ \text { atherosclerosis } & 767 & \text { chest compression } & 351 \\ \text { attitude } & 583 & \text { chip formation } & 253 \\ \text { attitude for elderly } & 515 & \text { chiropractic } & 325 \\ \text { autocorrelation } & 489 & \text { chronic obstructive pulmonary disease } & 91 \\ \text { autonomic dysfunction } & 885 & \text { cirrhosis } & 867 \\ \text { autotransfusion system } & 91 & \text { clinical cancer stage } & 497 \\ \text { auxiliary tool } & 123 & \text { clinical decision support system } & 63 \\ \text { average glandular dose } & 137 & \text { clinical results } & 263 \\ & 345 & \text { cognitive function } & 453 \\ \text { balance } & & \text { college students } & 435 \\ \text { berries } & 2715 & \text { complication } & 645 \\ \text { biceps brachii } & 339 & \text { computed radiography } & 427 \\ \text { biocompatible metals } & 505 & \text { computed tomography } & 253 \\ \text { bio-information architecture } & 867 & \text { concomitant fractures } & 877 \\ \text { biomechanics } & 387 & \text { cone beam computed tomography (CBCT) } & 333 \\ & 627 & \text { controlled mechanical ventilation } & 717 \\ & & & \end{array}$




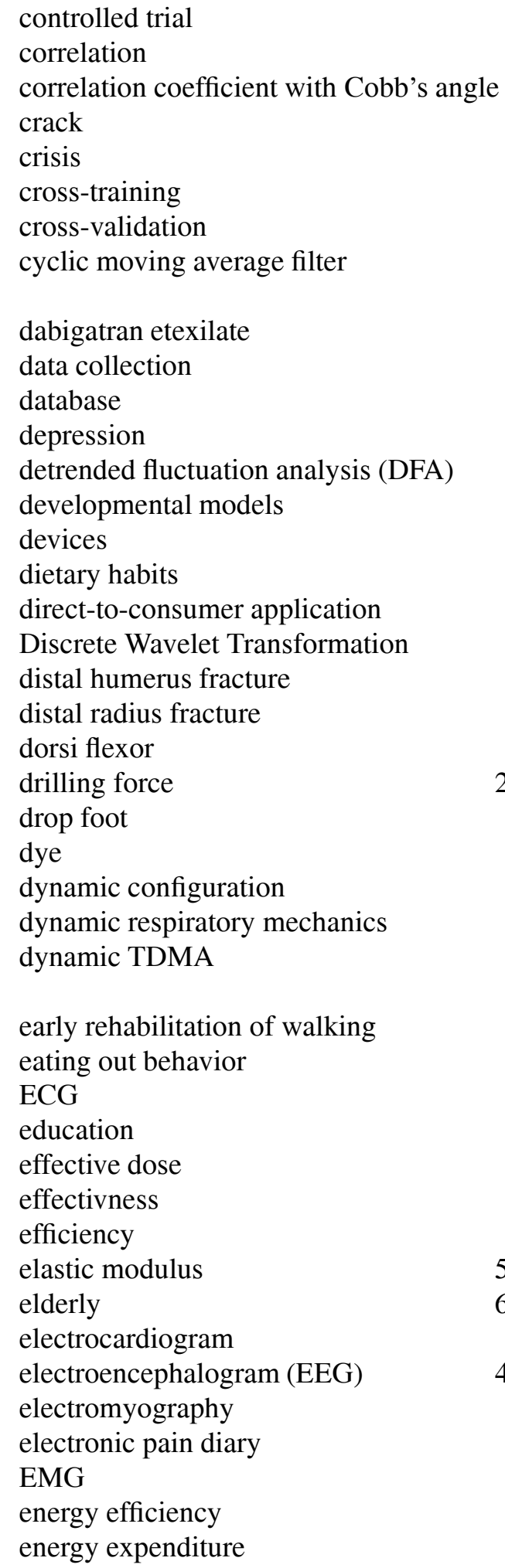

63 enoxaparin 901

403 exergame 199

741 factor analysis 379

547 fading time 427

759 fall 27

701 father's daughter 443

409 fatigue 395

feature selection $\quad 583$

901 female 129

689 female oedipus 443

137 finite element method 867

453 finite element simulation 741

885 fitting surface 573

847 flow rate 209

795 force 505

435 fruit farmer 359

817 functional electrical stimulation 751

701

909 gait analysis 751

877 gait feature 805

395 gait robotics 273

253,741 GC $\times$ GC-TOFMS 481

751 gender 129

339 genetic algorithm 835

387 genu varum 325

717 geriatric fractures $\quad 877$

167 glandular 345

goniometer 289

273 goniometter 751

435 GPS technology 689

651 gratitude disposition 459

847 grip force 617

403 GSM 561

123
317 haze

$\begin{array}{lll}573,785 & \text { health } & 677\end{array}$

677, 805 health care 77

835 health information management 317

419,835 health information technology 99

505 health messages 1

63 health perception 473

505 health technology assessment 729

167 healthcare 817

199 heart failure 37 
heart rate

heart rate variability

hemiplegia

high heels

Hip arthroplasty

hip arthroscopy

home care program

homecare informatics

horizon scanning

hospital based

hospital stay

human-centered design

Hybrid-ANFIS

\section{ICT}

image segmentation

imaging plate

impacted teeth

implementation

improvement

incisors

independent living

information platform

information technology

information visualization

infrared thermography

injury

integrated care

internet

intimal hyperplasia

IORT

IR array

ISO C-3D

isometric contraction

Kinect ${ }^{\circledR}$

kyphon

lab-on-a-chip system and LOC

lifestyle

lifestyle counseling

linear classifiers

liver

liver CT

lumbar lordosis angle
651 LWAs

561

91

751

309

901

667

91

99

729

729

877

77

835

561

895

427

333

147

297

785

677

27

547

657

243

369

677

597

209

729

137

115

617

27, 289

115

817

473

597

651

867

403

467

$\mathrm{M} / \mathrm{M} / 1$

561

maintenance $\quad 795$

male 129

malignancy $\quad 867$

mammography 701

manual lymph drainage (MLD) 309

MDCT

medical device

13

medical image retrieval

medication safety

memetic algorithm

mental arithmetic task

metabolic chamber

metabolic equivalent

methodologies

mHealth

M-health

microbubbles

microcalcifications detection

micro-CT

microRNA

middle age

middle school student

minimal invasive

mobile agent

mobile health

mobile phone

modeling

Monte Carlo simulation

mordant

motion artifacts

motion detection

motor level stimulation TENS

MRI segmentation

multi SVM

multiclass SVM classification

multilayer perceptron

musculoskeletal

musculoskeletal disorders (MSDs)

nail osteosynthesis

nanoindentation

narrative structure of the surface
909

785 


\begin{tabular}{|c|c|c|c|}
\hline \multirow{5}{*}{$\begin{array}{l}\text { narrative structure of the unconsciousness } \\
\text { navigation } \\
\text { network configuration slot } \\
\text { nonlinear modeling } \\
\text { nutrition education }\end{array}$} & 443 & \multirow{2}{*}{ primary health care } & 147 \\
\hline & 115 & & 667 \\
\hline & 167 & primary teeth & 785 \\
\hline & 515 & principal component analysis & 583,857 \\
\hline & 435 & $\begin{array}{l}\text { priority based ECG monitoring } \\
\text { privacy }\end{array}$ & $\begin{array}{l}167 \\
515\end{array}$ \\
\hline older adults & 189,657 & probabilistic neural network & 857 \\
\hline one-stage revision arthroplasty & 767 & process-steps design & 387 \\
\hline online & 597 & Props Pilates & 359 \\
\hline open hip surgery & 667 & prosthesis & 895 \\
\hline operating room & 795 & PSC & 667 \\
\hline optimization (ACO) & 775 & pullout test & 607 \\
\hline orthopaedic & 243 & & \\
\hline orthopedics & 741 & QR code & 99 \\
\hline oscillatory shear index & 209 & quadriceps muscle & 759 \\
\hline \multirow[t]{2}{*}{ osteoporosis } & 607 & qualitative content analysis & 147 \\
\hline & & quantitative & 147 \\
\hline pain & 359 & questionnaire & 147 \\
\hline parent artery & 209 & & \\
\hline particle swarm optimization (PSO) 531, & 775,857 & range of motion (ROM) & 289 \\
\hline patients & 317 & reading motivation & 379 \\
\hline pattern recognition & 651 & reassessment & 297 \\
\hline pediatric dentistry & 333 & rectal cancer & 497 \\
\hline pedicle screw fixation & 607 & reflective tape & 137 \\
\hline pelvic sacral angle & 467 & rehabilitation & $157,179,627,915$ \\
\hline people who inject drugs & 689 & rehabilitation robotics & 179 \\
\hline perceptual embodiment & 157 & relationship & 505 \\
\hline percutaneous cementation & 645 & relative speed & 427 \\
\hline personalization & 515 & remote monitoring & 63 \\
\hline persuasive technology & 189 & Resurfacing Arthroplasty (RA) & 263 \\
\hline physician-manufacturer communication & 297 & review of literature & 667 \\
\hline Phytolacca americana $\mathrm{L}$. & 339 & revision arthroplasty & 895 \\
\hline plantar flexor & 395 & revision surgery & 645 \\
\hline plantar pressure & 805 & RFID & 795 \\
\hline PMMA & 607 & risk & 817 \\
\hline polishing & 573 & rural & 597 \\
\hline polycystic ovary syndrome & 857 & rural areas & 147 \\
\hline positive identity & 459 & & \\
\hline post-operative & 63 & sacral slope & 467 \\
\hline post-purchase & 297 & sacrificial piety & 443 \\
\hline postural sway & 395 & safety & 795 \\
\hline posture & 325 & sand blasting & 573 \\
\hline PPG & 409 & satisfaction & 77 \\
\hline practice & 1 & scale development & 379 \\
\hline premature ventricular contractions & 651 & scanning electron microscopy & 741 \\
\hline
\end{tabular}


scoliosis evaluation index

screw loosening

second language learning

self efficacy

self integrity

self-management

self-test

seminal

sensor

sensor placement

simulation

Sit-to-Stand strategy

skull bone region

sleep stages

smart home

smartphone

smartphone application

SMS

Sobel operator

social support

solid phase micro-extraction (SPME)

spinal deformity

spine biomechanics

spine degeneration

spine fracture

stability

stent

storage temperature

strength

stress response

STULONG dataset

supernumerary teeth

supine stepping

support vector machine

surface electromyography

surface roughness

surgical hip dislocation

sweat

swelling

systematic review

systemical program
225 Taiwan

607 TAM

379 technology

147,847

489

489

677

817

531

657,677

505

627

627

775

885

657

351

547,847

1

701

489

481

225

607

607

607

359

209

427

759

419

583

333

273

531, 857

617

253

667

481

309

189

497

template matching $\quad 895$

thermal necrosis 243

thromboembolism prophylaxis $\quad 901$

tibial plateau fracture $\quad 115$

tilt table 179

TKA 129

total hip arthroplasty $\quad 123$

total hip resurfacing 263

total knee arthroplasty

$123,645,767$

transcutaneous electrical nerve stimulation

(TENS)

395,157

triceps brachii muscle

ultrasonic vibrations 253

ultrasonically-assisted drilling $\quad 243$

ultrasound 37

ultrasound therapy $\quad 309$

under-graduate psychiatry education

innovations

847

vestibular

915

video consultation $\quad 147$

virtual reality 27, 915

visual input 395

volatile organic compounds (VOCs) 481

wall shear stress 209

web services 677

wireless body sensor network $\quad 167$

wireless monitoring $\quad 805$

women patients 497

wound secretion 901

x-ray 895

$\begin{array}{ll}\text { Yangsaeng } & 473\end{array}$ 